\title{
Artificial intelligence algorithm to predict the need for critical care in prehospital emergency medical services
}

Da-Young Kang ${ }^{1+}$, Kyung-Jae $\mathrm{Cho}^{2 \dagger}{ }^{+}$, Oyeon Kwon ${ }^{2+}$, Joon-myoung Kwon ${ }^{1,3^{*}}$ (D) Ki-Hyun Jeon ${ }^{1,4}$, Hyunho Park ${ }^{2}$, Yeha Lee ${ }^{2}$, Jinsik Park ${ }^{4}$ and Byung-Hee $\mathrm{Oh}^{4}$

\begin{abstract}
Background: In emergency medical services (EMSs), accurately predicting the severity of a patient's medical condition is important for the early identification of those who are vulnerable and at high-risk. In this study, we developed and validated an artificial intelligence (Al) algorithm based on deep learning to predict the need for critical care during EMS.
\end{abstract}

Methods: We conducted a retrospective observation cohort study. The algorithm was established using development data from the Korean national emergency department information system, which were collected during visits in real time from 151 emergency departments (EDs). We validated the algorithm using EMS run sheets from two EDs. The study subjects comprised adult patients who visited EDs. The endpoint was critical care, and we used age, sex, chief complaint, symptom onset to arrival time, trauma, and initial vital signs as the predicted variables.

Results: The number of patients in the development data was 8,981,181, and the validation data comprised 2604 EMS run sheets from two hospitals. The area under the receiver operating characteristic curve of the algorithm to predict the critical care was 0.867 (95\% confidence interval, [0.864-0.871]). This result outperformed the Emergency Severity Index (0.839 [0.831-0.846]), Korean Triage and Acuity System (0.824 [0.815-0.832]), National Early Warning Score (0.741 [0.734-0.748]), and Modified Early Warning Score (0.696 [0.691-0.699]).

Conclusions: The Al algorithm accurately predicted the need for the critical care of patients using information during EMS and outperformed the conventional triage tools and early warning scores.

Keywords: Emergency medical service, Triage, Artificial intelligence, Deep learning

\section{Introduction}

An important objective of emergency medical services (EMSs) is to provide appropriate prehospital

\footnotetext{
* Correspondence: kwonjm@sejongh.co.kr

${ }^{\dagger}$ Da-Young Kang, Kyung-Jae Cho and Oyeon Kwon contributed equally to this work.

${ }^{1}$ Artificial Intelligence and Big Data Research Center, Sejong Medical

Research Institute, 20, Gyeyangmunhwa-ro, Gyeyang-gu, Incheon, Republic of Korea

${ }^{3}$ Department of Emergency Medicine, Mediplex Sejong Hospital, 20, Gyeyangmunhwa-ro, Gyeyang-gu, Incheon, Republic of Korea Full list of author information is available at the end of the article
}

management and transfer to the relevant emergency department (ED) based on a patient's status [1]. Several prognosis prediction tools have been developed for EMS but are limited to specific situations, such as trauma [2]. Although some efforts have been made to apply existing ED triage tools and early warning scores to EMSs, these tools have so far performed unsatisfactorily [3].

In EMS, accurately predicting the need for critical care is important for the early identification of the vulnerability and high-risk of patients, and for deciding the most appropriate management during transfer [4]. If the

(C) The Author(s). 2020 Open Access This article is licensed under a Creative Commons Attribution 4.0 International License, which permits use, sharing, adaptation, distribution and reproduction in any medium or format, as long as you give appropriate credit to the original author(s) and the source, provide a link to the Creative Commons licence, and indicate if changes were made. The images or other third party material in this article are included in the article's Creative Commons licence, unless indicated otherwise in a credit line to the material. If material is not included in the article's Creative Commons licence and your intended use is not permitted by statutory regulation or exceeds the permitted use, you will need to obtain permission directly from the copyright holder. To view a copy of this licence, visit http://creativecommons.org/licenses/by/4.0/. The Creative Commons Public Domain Dedication waiver (http://creativecommons.org/publicdomain/zero/1.0/) applies to the data made available in this article, unless otherwise stated in a credit line to the data. 
patient is expected to require critical care, the EMS technician must pass through the nearest low-level ED to a high-level ED [5]. Accurate tools for predicting prognosis are important for communication between the prehospital EMS technician and hospital medical staff to provide online medical directions and prepare inhospital management [6, 7].

The goal of this study was to develop and validate an artificial intelligence (AI) algorithm based on deep learning to predict the need for critical care of patients in EMSs accurately. Deep learning could overcome the limitations of conventional statistical methods and has recently achieved state-of-the-art performance in several domains, including medical imaging and outcome prediction [8-10]. To the best of our knowledge, this study is the first to predict severity in EMS using an AI algorithm.

\section{Methods}

\section{Study design and setting}

This was a multicenter retrospective cohort study, not a blind study. Furthermore, the study was entirely separated between development and external validation data. To establish the AI algorithm, we used the Korean national emergency department information system (NEDIS), which collects all patient visits in real time from 151 EDs in Korea. To externally validate our model, EMS run sheets from patients who visited two EDs were used. Specifically, the EMS run sheets contain information on when patients were contacted by an EMS. The run sheets were saved as electronic medical records. The sample size of the validation dataset was determined using an accurate algorithm in a previous study [11].

The data comprised age, sex, chief complaint, time from symptom onset to visit (or EMS contact), trauma, initial vital signs (systolic blood pressure, diastolic blood pressure, heart rate, respiratory rate, and body temperature), and mental status; these data were used as the predictor variables. The endpoint of this study was critical care (admission to intensive care unit). For the stabilized training, the input variables were normalized with a z-score.

The institutional review boards of Sejong General Hospital (2019-0212) and Mediplex Sejong Hospital (2019-049) approved this study protocol and waived the need for informed consent because of the impracticality and minimal harm involved.

\section{Selection of participants}

The study participants were adult patients (aged $\geq 18$ years) who visited EDs. From the development data (NEDIS), we selected adult patients who visited EDs between January 2014 and December 2016. Moreover, we selected patients who visited two EDs using EMSs between September 2018 and February 2019 as the test data. We excluded subjects who were declared dead on arrival and those for whom data were missing, as shown in Fig. 1.

\section{Development of Al algorithm based on deep learning}

To establish our algorithm, development data (NEDIS) were utilized. To classify the presence of critical care needs, we used feedforward networks (5 hidden layers, 89 nodes, and batch normalization $[12,13])$, which train the output using the softmax classifier. We applied a dropout rate of 0.5 at each layer for regularization and a rectified linear unit was used for the activation function. The Adam optimizer was used to improve the efficiency of optimization, while the cross-entropy loss function was used to minimize the prediction loss based on a supervised learning. In addition, we used TensorFlow (the Google Brain Team, Mountain View, United States) as the backend [14]. The calibration plot and Brier score are described in a supplemental figure.

\section{Performance test of Al algorithm and comparison with conventional methods}

We compared the performance of the algorithm in terms of predicting critical care with those of the Emergency Severity Index (ESI), Korean Triage and Acuity System (KTAS), Modified Early Warning Score (MEWS), and National Early Warning Score (NEWS). The ESI is a globally used five-level ED triage algorithm, initially developed in 1999 [15, 16]. It is based on the severity of patients' healthcare problems and the number of resources that is anticipated to require. KTAS was developed in 2012 based on the Canadian Triage and Acuity Scale and has been used nationwide as a triage since 2016 in Korea $[11,17,18]$. KTAS is a five-level ED triage algorithm that considers symptoms, pain, and physiological values. Three medical staff members with more than 5 years of experience in clinical practice in an ED participated in this study. They decided the ESI and KTAS levels with information from the EMS run sheets for patients in the test data. Conflicting results were decided by discussion.

MEWS is a widely used tool for predicting severity and deterioration, and is calculated using systolic blood pressure, heart rate, respiratory rate, body temperature, and mental status [19]. NEWS was developed in the United Kingdom. It is a popular aggregated scoring system that considers respiratory rate, oxygen saturation, temperature, systolic blood pressure, heart rate, and mental status [20]. The MEWS and NEWS scores have been well-validated and used globally. In previous studies, some efforts have been made to apply these early warning scores to EMSs [21]. We calculated the MEWS and NEWS scores based on information from the EMS 


\section{Development data}

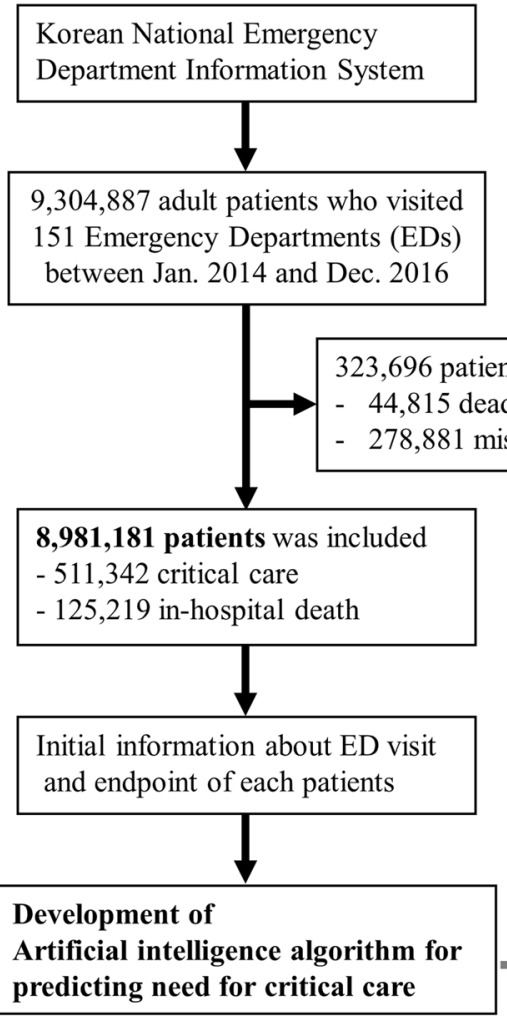

\section{Test data}

\section{8 adult patients}

who visited 2 EDs using EMS

between Sep 2018 and Feb 2019

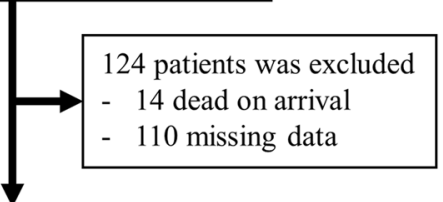

2604 patients was included

- 319 critical care

- 30 in-hospital death

Information about EMS run sheets

and endpoint of each patients

Accuracy test of developed algorithm

using test data

Fig. 1 Study flowchart. Legends: ED: emergency department; EMS: emergency medical service

run sheets. The EMS run sheets comprise data at the time of first contact of EMSs with each patient.

We validated the developed algorithm using exclusively divided test data. The performance measures were taken as the area under the receiver operating characteristic curve (AUC), sensitivity, specificity, positive predictive value (PPV), negative predictive value (NPV), and F1 score. The AUC is a frequently used metric and shows the sensitivity against 1 -specificity [22]. Based on previous studies, we used levels $1-2$, levels $1-2$, points $3-14$, and points 5-20 to predict the critical care with the ESI, KTAS, MEWS, and NEWS, respectively [11, 17-21]. When evaluating the continuous score predicted by the AI algorithm, we fixed the sensitivity as 0.8. Furthermore, we evaluated the $95 \%$ confidence interval using bootstrapping (10,000 times resampling with replacement) [23]. We used the ROCR package in $\mathrm{R}$ ( $\mathrm{R}$ Development Core Team, Vienna, Austria) for these analyses.

Combining the $\mathrm{Al}$ algorithm and conventional triage tools With the aim of developing a high-performance algorithm, we combined the AI algorithm with conventional triage tools. This method is called ensemble [24]. A major limitation of the ESI and KTAS, as reported by previous studies, is the decreasing accuracy attributed to patients at mid-level, such as level 3. We applied the AI algorithm for patients at level 3 for each ESI and KTAS, and validated the performance of the two ensemble models (AI+ESI and AI+KTAS). For this, patients at levels 1 and 2 were predicted to be critical while patients at levels 4 and 5 were predicted to be noncritical. The AI algorithm only evaluated the patients at level 3 .

\section{Results}

In the development data, in total, 9,304,887 ED visits to 151 hospitals were included in the NEDIS. We excluded 323,696 visits because of the exclusion criteria: 44,815 were declared dead on arrival, while data were missing for 278,881 visits. No significant differences in the predictor variables were observed between the included and excluded study subjects due to the missing variables. Thus, the study subjects included 8,981,181 ED patients; 511,342 ended up in critical care (5.7\%) and 125,219 (1.4\%) died in hospital.

In the case of the test data, after excluding 124 patients (14 dead on arrival and 110 missing data), validation of the $\mathrm{AI}$ algorithm in EMSs was performed using 2604 patients from two hospitals, whose endpoints were 319 in critical care $(12.3 \%)$ and 30 of whom died in hospital (1.2\%). 
The baseline characteristics of the development and test data are shown in Table 1. These two data were exclusively divided, and their characteristics are significantly different.

As shown in Fig. 2, the AUC of the AI algorithm was 0.867 (95\% confidence interval [0.864-0.871]), which outperformed the ESI (0.839), KTAS (0.824), NEWS (0.741), and MEWS (0.696). Furthermore, the ensemble algorithm AI + ESI (0.923 [0.920-0.926]) significantly outperformed the other ensemble algorithm AI + KTAS (0.909 [0.9060.912]), AI algorithm, and other conventional methods.

\section{Discussion}

This study demonstrated that the AI algorithm accurately predicted the need for critical care in a prehospital EMS situation.
Predicting the need for critical care is important for selecting the destination ED and for providing the appropriate management during transfer $[4,5]$. In addition, tools for accurately predicting the prognosis and treatment are important to communicate between the prehospital EMS technician and hospital medical staff $[6,7]$. However, most triage tools in prehospital situations were developed for trauma patients only, and there is no generalized tool that covers all EMS situations [2, 25]. Although the conventional triage tools of EDs have been applied to predict the need for critical care at prehospital situations [21, 26, 27], they showed an unsatisfactory performance in predicting prognosis.

The important finding of this study is that the predictive performance of the AI model based on deep learning is superior to those of the conventional triage tools and

Table 1 Baseline characteristics ${ }^{\mathrm{a}}$

\begin{tabular}{|c|c|c|c|}
\hline Characteristics & $\begin{array}{l}\text { Development data } \\
(\boldsymbol{n}=8,981,181)\end{array}$ & $\begin{array}{l}\text { Test data } \\
(\boldsymbol{n}=2604)\end{array}$ & $p$-value \\
\hline \multicolumn{4}{|l|}{ Data } \\
\hline Data type & National Emergency Department Infromation System (NEDIS) & Emergency Medical Service (EMS) Run Sheets & \\
\hline Data source & Emergency department visit data & EMS run sheets & \\
\hline Data period & 1 January 2014-30 June 2016 & 1 September 2018-28 February 2019 & \\
\hline Age & $49.9 \pm 18.9$ & $61.5 \pm 18.6$ & $<0.001$ \\
\hline Female, No.(\%) & $4,511,654(50.2 \%)$ & $1411(54.2 \%)$ & $<0.001$ \\
\hline \multicolumn{4}{|c|}{ Initial vital signs, mean \pm SD } \\
\hline Systolic BP (mmHg) & $131.2 \pm 23.3$ & $132.0 \pm 24.6$ & 0.271 \\
\hline Diastolic BP (mmHg) & $79.3 \pm 13.9$ & $83.7 \pm 17.4$ & $<0.001$ \\
\hline Heart rate (/min) & $83.8 \pm 16.2$ & $85.5 \pm 20.5$ & $<0.001$ \\
\hline Respiratory rate (/min) & $19.6 \pm 2.7$ & $17.7 \pm 3.3$ & $<0.001$ \\
\hline Body temperature $\left({ }^{\circ} \mathrm{C}\right)$ & $36.7 \pm 0.7$ & $36.7 \pm 0.8$ & $<0.001$ \\
\hline \multicolumn{2}{|l|}{ Mental status, No.(\%) } & & $<0.001$ \\
\hline Alert & $8,674,058(96.6 \%)$ & $2513(96.5 \%)$ & \\
\hline Reacting to voice & $161,624(1.8 \%)$ & $20(0.8 \%)$ & \\
\hline Reacting to pain & $113,192(1.3 \%)$ & $46(1.8 \%)$ & \\
\hline Unresponsive & $32,310(0.3 \%)$ & $25(1.0 \%)$ & \\
\hline Trauma, No.(\%) & $2,536,815(28.2 \%)$ & $550(21.1 \%)$ & $<0.001$ \\
\hline \multicolumn{3}{|c|}{ Symptome onset to visit (contact) time, No.(\%) } & $<0.001$ \\
\hline$-24 h$ & $5,394,527(60.1 \%)$ & $2105(80.8 \%)$ & \\
\hline $24 \mathrm{~h}-72 \mathrm{~h}$ & $2,666,179(29.7 \%)$ & $448(17.2 \%)$ & \\
\hline $72 \mathrm{~h}-7$ Days & $536,525(6.0 \%)$ & $38(1.5 \%)$ & \\
\hline 7 Days-30 Days & $258,641(2.9 \%)$ & $12(0.5 \%)$ & \\
\hline 30 Days- & $125,312(1.4 \%)$ & $1(0.0 \%)$ & \\
\hline \multicolumn{4}{|l|}{ Outcomes, No.(\%) } \\
\hline Critical care & $511,342(5.7 \%)$ & $319(12.3 \%)$ & 0.006 \\
\hline In-hospital mortality & 125,219 (1.4\%) & $30(1.2 \%)$ & $<0.001$ \\
\hline Hospitalization & 2,443,994 (27.1\%) & 1003 (38.5\%) & $<0.001$ \\
\hline
\end{tabular}

aP denotes blood pressure

${ }^{\mathrm{b}}$ The alternative hypothesis for this $p$-value was that there is a difference between the development and test data group for each variable 


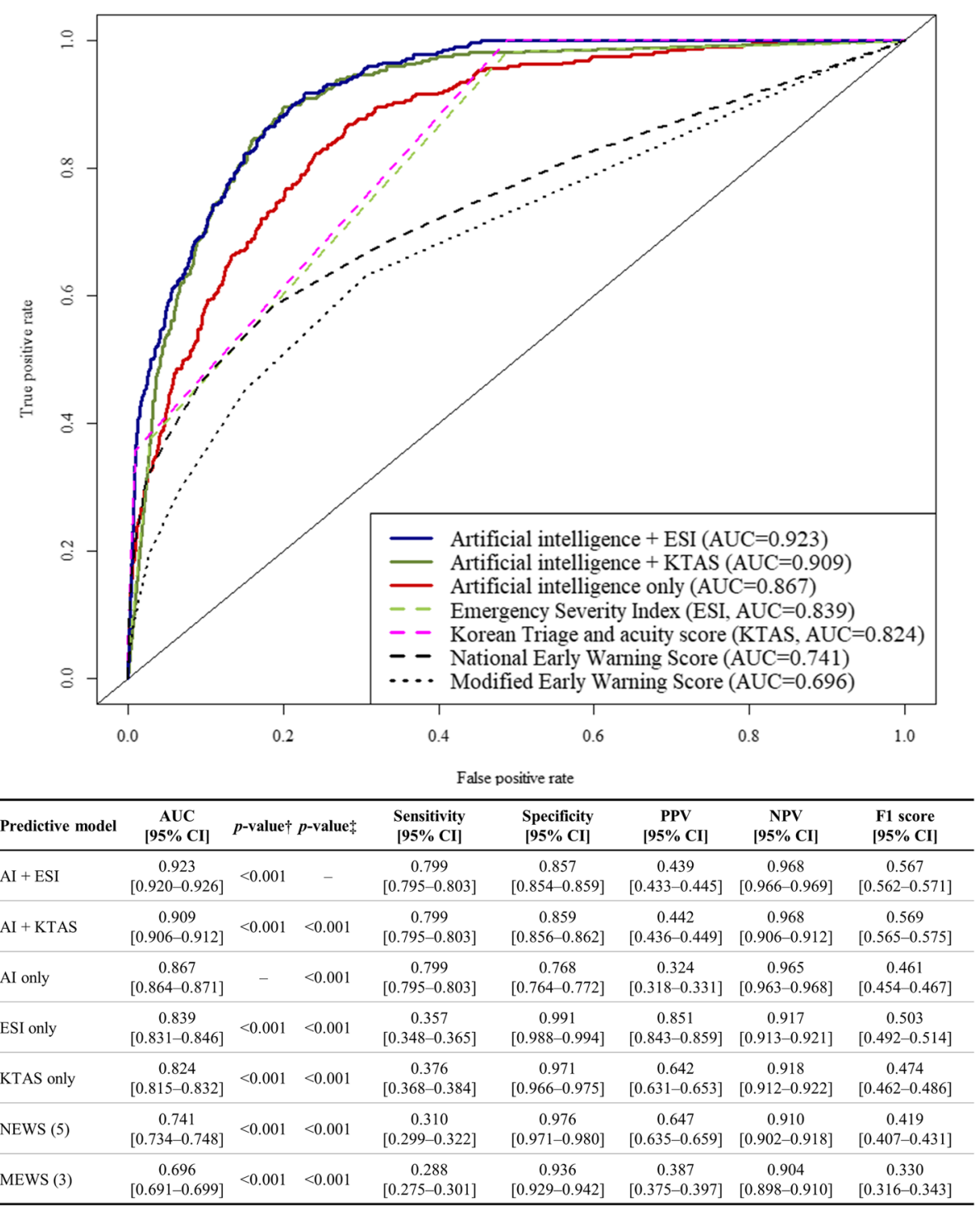

Fig. 2 Receiver operating characteristics curve for predicting critical care Legends: *Al: artificial intelligence; AUC: area under the receiver operating characteristics curve; Cl: confidence interval; ESI: Emergency Severity Index, KTAS: Korean triage and acuity system; MEWS: modified early warning score; NEWS: national early warning score; NPV: negative predictive value; PPV: positive predictive value. †The alternative hypothesis for this $p$-value was that there is a difference between the artificial intelligence algorithm and the other predictive methods. ‡The alternative hypothesis for this $p$-value was that there is a difference between the ensemble model, combining artificial intelligence and the ESI, and the other predictive methods

scoring systems. In addition, three ED medical staff members were involved in deciding the level of triage with EMS run sheets. Interestingly, the accuracy of the AI algorithm was better than the accuracy of the decision of the expert medical staff. The AI algorithm performs automatic calculations based on basic information and does not require expert judgment and medical experience.

Deep learning can obtain a high performance without prior knowledge to train the model; thus, indicating that deep learning somehow automatically learns the feature relationship among input variables. In our previous study, we developed an AI algorithm based on deep learning for predicting the critical care of patients in an ED [11]. From the previous study, we found that conventional statistical methods such as logistic regression may have difficulty in determining the relationship among input variables $[10,28,29]$. As a large number of input variables were utilized, the dimensionality of the input increased. This somehow indicates that the process of feature extraction by humans should be required and effort should be made to determine the relationship between input variables. 
Meanwhile, deep learning includes feature learning, which allows the model to automatically learn the relationships and characteristics between input variables required to perform a task [30]. As shown in our previous studies, deep learning could be used to understand the connection between features and outperformed conventional and other machine learning methods $[9,11,31]$. It is important to note that feature learning is not designed by humans in deep learning. As this process evolves automatically, it will be easier and more effective to identify intricate structures in high-dimensional data without information loss, and will result in end-to-end learning, which requires little engineering by humans. Finally, it can be easily and quickly applied to other tasks [30].

In addition, one of the well-known concepts in the use of deep learning is the importance of the amount of data. The accumulation of numerous data for decades advanced the performance improvement of deep learning. Likewise, the performance of the model based on deep learning depends greatly on the amount of data. In this study, we used the NEDIS data, which comprise millions of data. We believe that this amount of accumulated data would be more suitable for deep learning than other approaches. Moreover, we only used the initial vital signs for patients (assuming that it would be difficult to measure vital signs several times during transport). We considered the simple DNN model as more suitable than LSTM. Because the LSTM is based on sequential information.

The prevention of overfitting into a single hospital is an important issue. Further, it is crucial to verify whether the model was overfitted to a specific environment. Thus, the acquisition of external validation data is important. Wolpert explains this in the "No Free Lunch" theorem: If optimized in one situation, an algorithm cannot produce good results in other situations [32]. In this study, the development (ED) and test data (EMS) were exclusively divided. More specifically, the model was evaluated on the external dataset and could possibly avoid overfitting in one environment.

A major limitation of conventional triage tools is their low accuracy at the middle level $[18,33,34]$. As shown in Fig. 3, at level 3 triages, the population were mixed as critical care and non-critical care patients. If the patients at level 3 can be distinguished, we consider that the accuracy of predicting the outcome will increase. Therefore, we made effort to apply the algorithm for patients at level 3.

In this study, we developed the ensemble algorithm to evaluate level 3 patients and confirmed a higher

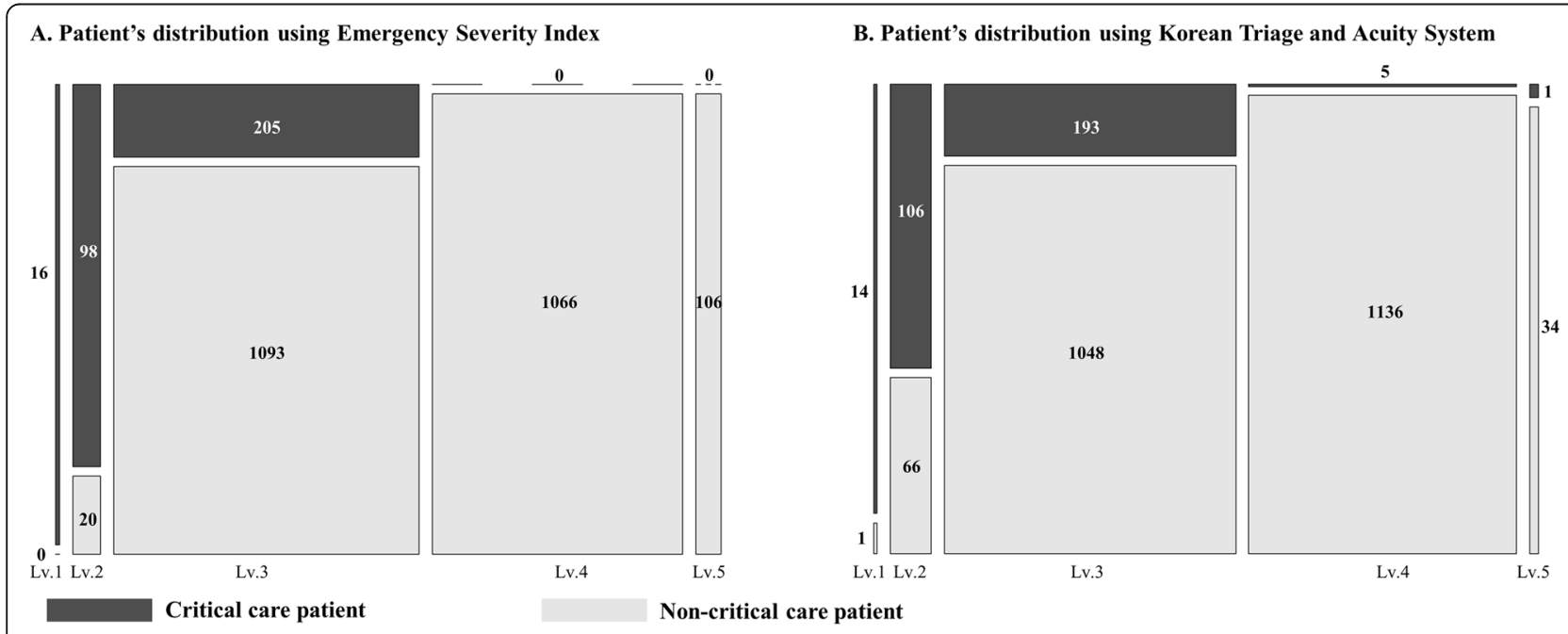

C. Performance to predict need for critical care with Emergency Severity Index and Korean Triage and Acuity System

\begin{tabular}{lcccc}
\hline & \multicolumn{4}{c}{ Emergency Severity Index } \\
\cline { 2 - 5 } & Level 1 & Level 1-2 & Level 1-3 & Level 1-4 \\
\hline Sensitivity & 0.050 & 0.357 & 1.000 & 1.000 \\
\hline Specificity & 1.000 & 0.991 & 0.513 & 0.046 \\
\hline Positive Predictive Value & 1.000 & 0.851 & 0.223 & 0.128 \\
\hline Negative Predictive Value & 0.883 & 0.917 & 1.000 & 1.000 \\
\hline F1 score & 0.095 & 0.503 & 0.365 & 0.227 \\
\hline
\end{tabular}

\begin{tabular}{lcccc}
\hline & \multicolumn{4}{c}{ Korean Triage and Acuity System } \\
\cline { 2 - 5 } & Level 1 & Level 1-2 & Level 1-3 & Level 1-4 \\
\hline Sensitivity & 0.044 & 0.376 & 0.981 & 0.997 \\
\hline Specificity & 1.000 & 0.971 & 0.512 & 0.015 \\
\hline Positive Predictive Value & 0.933 & 0.642 & 0.219 & 0.124 \\
Negative Predictive Value & 0.882 & 0.918 & 0.995 & 0.971 \\
\hline F1 score & 0.084 & 0.474 & 0.358 & 0.221 \\
\hline
\end{tabular}

Fig. 3 Patient distributions and performance using conventional triage systems 
performance with the AI+ESI algorithm. It is interesting to note that the combination of the expert opinion (ESI level) and the AI algorithm exhibits a more accurate performance. These results provide an opportunity to solve the problem for researchers in other medical fields. For example, in previous studies of urology, AI algorithms have been applied for the prediction of prostate biopsy results and the recurrence-free probability of bladder cancer [35]. In addition, the in-hospital and long-term mortalities of patients with cardiovascular diseases have been predicted using AI algorithms in several previous studies.

Our study has several limitations. First, deep learning is considered a black box. Although we can fit the AI algorithm based on deep learning, it is difficult to fully understand how the model predicts critical care. In addition, contrary to traditional methods, such as XGboost or CatBoost that can present uncertainty measures (e.g., 95\% confidence interval), deep learning has greater difficulty in quantifying the uncertainty measures. In this paper, to describe the uncertainty information, we attempted to quantitatively measure the uncertainty as much as possible through bootstrapping [23]. In fact, recent attempts have been made to explain deep learning and measuring uncertainty, which will be our next area of study [36, 37]. Second, as this study was conducted in only two hospitals in Korea, it is necessary to validate the model for patients in EMSs in greater populations or other countries.

We developed a high-performance algorithm by combining an AI algorithm and a conventional triage tool. Despite several limitations, deep learning achieved a high predictive performance in several medical domains. Further, the deep learning algorithm can be developed more easily than a machine learning method. Based on our methodologies and results, other researchers can develop algorithms for their own groups of patients and situations. Additionally, medical researchers could investigate the applicability and future development of deep learning in various domains of medicine. For example, using this algorithm, the need for the critical care of patients could be predicted during EMS situations, and the destination hospital could be optimized by considering the predicted critical care needs and hospital's situation (e.g., ED overcrowding, ICU capacity, and critical care availability). Moreover, the predictor variables in this algorithm were simple and could be used via a wearable device and information from a patient or their family. Because of this, patients with severe underlying diseases could be monitored daily while they are living at home regarding their needs for critical care, and could be referred to hospital earlier if they exhibit deterioration.

\section{Conclusion}

In this study, a triage using an AI algorithm accurately predicted the need for critical care of patients using information during EMS situations, and outperformed the conventional triage tools and early warning scores. The results showed the potential of AI for EMSs, which will be a useful and fast tool to identify vulnerable patients and help precise decision-making in daily practice.

\section{Supplementary information}

Supplementary information accompanies this paper at https://doi.org/10. 1186/s13049-020-0713-4.

Additional file 1. Supplemental material. Brier scores and calibration plot.

\section{Abbreviations}

Al: Artificial intelligence; AUC: Area under the receiver operating characteristic curve; DNN: Deep neural network; ED: Emergency department; EMS: Emergency medical service; ESI: Emergency Severity Index; ICU: Intensive care unit; KTAS: Korean triage and acuity system; MEWS: Modified early warning score; NEDIS: National emergency department information system; NEWS: National early warning score; NPV: Negative predictive value; PPV: Positive predictive value; ReLU: Rectified linear unit

\section{Acknowledgements}

None.

\section{Authors' contributions}

DYK contributed to the study idea and design, and data collection, undertook the data analysis, and contributed to the subsequent drafts. OK and KJC prepared and verified the clinical coding and performed the data analysis. $\mathrm{HP}$ and $\mathrm{YL}$ performed the data analysis. $\mathrm{KHJ}$, JP, and $\mathrm{BHO}$ contributed to the data collection and revised the manuscript. JMK is the principal investigator and contributed to the study idea and design, interpreted the data, prepared and verified the clinical coding, undertook the data analysis, wrote the first draft, and contributed to the subsequent drafts. The authors read and approved the final manuscript

\section{Authors' information}

None

\section{Funding}

This work was supported by the Technology Innovation Program (2000486, Common data model based intelligent algorithm network environment platform for precision clinical decision support system), funded by the Ministry of Trade, Industry \& Energy (Korea).

\section{Availability of data and materials}

The data underlying this study belong to the National Emergency Medical Center (NEMC) of Korea. NEMC provides de-identified national emergency department information system data to researchers for nonprofit academic research. Any researchers who propose a study subject and plans with a standardized proposal form, and are approved by the NEMC review committee on research support can access the raw data. Details of this process and a provision guide are now available at the NEMC website (https://dw.nemc. or.kr) or contact point of the NEMC review committee (skko@nmc.or.kr). The authors accessed the data used in this study in the same manner that they expect future researchers to do so and did not receive special privileges from the NEMC of Korea.

\section{Ethics approval and consent to participate}

The institutional review boards of Sejong General Hospital (2019-0212) and Mediplex Sejong Hospital (2019-049) approved this study protocol and waived the need for informed consent because of the impracticality and minimal harm involved.

Consent for publication Not applicable. 


\section{Competing interests}

VUNO provided support in the form of salaries for the authors (Kyung-Jae Cho, Oyeon Kwon, Hyunho Park, and Yeha Lee), but did not have any additional role in the study design, data collection and analysis, decision to publish, or preparation of the manuscript. Dr. Yeha Lee is the co-founder and stakeholder in VUNO Inc., a medical artificial intelligence company. Mr. Kyung-Jae Cho, Oyeon Kwon, and Hyunho Park, are employees of VUNO Inc. Mr. There are no patents, products in development, or marketed products to declare. This does not alter our adherence to PLOS ONE policies on sharing data and materials.

\section{Author details}

${ }^{1}$ Artificial Intelligence and Big Data Research Center, Sejong Medical Research Institute, 20, Gyeyangmunhwa-ro, Gyeyang-gu, Incheon, Republic of Korea. ${ }^{2}$ VUNO, Seoul, South Korea. ${ }^{3}$ Department of Emergency Medicine, Mediplex Sejong Hospital, 20, Gyeyangmunhwa-ro, Gyeyang-gu, Incheon, Republic of Korea. ${ }^{4}$ Division of Cardiology, Cardiovascular Center, Mediplex Sejong Hospital, Incheon, South Korea.

\section{Received: 12 December 2019 Accepted: 21 February 2020}

Published online: 04 March 2020

\section{References}

1. Moore L. Measuring quality and effectiveness of prehospital ems. Prehospital Emerg Care. 1999;3:325-31..

2. Baxt WG, Jones G, Fortlage $D$. The trauma triage rule: a new, resource-based approach to the prehospital identification of major trauma victims. Ann Emerg Med. 1990;19:1401-6.

3. Lidal IB, Holte HH, Vist GE. Triage systems for pre-hospital emergency medical services - a systematic review. Scand J Trauma Resusc Emerg Med. 2013;21:28.

4. Seymour CW. Prediction of critical illness during out-of-hospital emergency care. JAMA. 2010;304:747.

5. Kahn JM, Branas CC, Schwab CW, Asch DA. Regionalization of medical critical care: what can we learn from the trauma experience? Crit Care Med. 2008;36:3085-8.

6. Evans SM, Murray A, Patrick I, Fitzgerald M, Smith S, Andrianopoulos N, et al. Assessing clinical handover between paramedics and the trauma team. Injury. 2010:41:460-4

7. Dojmi Di Delupis F, Pisanelli P, Di Luccio G, Kennedy M, Tellini S, Nenci N, et al. Communication during handover in the pre-hospital/hospital interface in Italy: from evaluation to implementation of multidisciplinary training through high-fidelity simulation. Intern Emerg Med. 2014;9:575-82.

8. Gulshan V, Peng L, Coram M, Stumpe MC, Wu D, Narayanaswamy A, et al. Development and validation of a deep learning algorithm for detection of diabetic retinopathy in retinal fundus photographs. Jama. 2016;304:649-56.

9. Kwon J, Jeon K-H, Kim HM, Kim MJ, Lim S, Kim K-H, et al. Deep-learningbased out-of-hospital cardiac arrest prognostic system to predict clinical outcomes. Resuscitation. 2019;139:84-91.

10. Breiman L. Statistical Modeling : The Two Cultures. 2011;16:199-215.

11. Kwon J, Lee $Y$, Lee $Y$, Lee S, Park H, Park J. Validation of deep-learning-based triage and acuity score using a large national dataset. PLoS One. 2018;13(10): e0205836. https://doi.org/10.1371/journal.pone.0205836. eCollection 2018.

12. Schalkoff RJ. Pattern recognition - statistical, structural and neural approaches; 1992

13. Ioffe S, Szegedy C. Batch Normalization: Accelerating Deep Network Training by Reducing Internal Covariate Shift; 2015.

14. Abadi M, Barham P, Chen J, Chen Z, Davis A, Dean J, et al. TensorFlow: A System for Large-Scale Machine Learning TensorFlow: A system for largescale machine learning. In: 12th USENIX Symp Oper Syst Des Implement (OSDI '16); 2016. p. 265-84.

15. Gilboy N, Tanabe P, Travers D. Rosenau AM. Emergency Severity Index (ESI): A Triage Tool for Emergency Department Care, Version 4: Implementation Handbook 2012 Edition; 2012.

16. Mistry B, Stewart De Ramirez S, Kelen G, PSK S, Balhara KS, Levin S, et al. Accuracy and Reliability of Emergency Department Triage Using the Emergency Severity Index: An International Multicenter Assessment. Ann Emerg Med. 2018;71:581-7 e3.

17. Kwon H, Kim YJ, Jo YH, Lee JH, Lee JH, Kim J, et al. The Korean triage and acuity scale: associations with admission, disposition, mortality and length of stay in the emergency department. Int J Qual Heal Care. 2018.
18. Lee B, Kim DK, Park JD, Kwak YH. Clinical considerations when applying vital signs in pediatric Korean triage and acuity scale. J Korean Med Sci. 2017;32: 1702.

19. Subbe CP, Kruger M, Rutherford P, Gemmel L. Validation of a modified early warning score in medical admissions. QJM. 2001;94:521-6.

20. Smith GB, Prytherch DR, Meredith P, Schmidt PE, Featherstone PI. The ability of the National Early Warning Score (NEWS) to discriminate patients at risk of early cardiac arrest, unanticipated intensive care unit admission, and death. Resuscitation. 2013;84:465-70.

21. Williams TA, Tohira H, Finn J, Perkins GD, Ho KM. The ability of early warning scores (EWS) to detect critical illness in the prehospital setting: a systematic review. Resuscitation. 2016;102:35-43

22. Jouffroy R, Saade A, Ellouze S, Carpentier A, Michaloux M, Carli P, et al. Prehospital triage of septic patients at the SAMU regulation: comparison of qSOFA, MRST, MEWS and PRESEP scores. Am J Emerg Med. 2018:36:820-4.

23. Carpenter J, Bithell J. Bootstrap confidence intervals: when, which, what? A practical guide for medical statisticians. Stat Med. 2000;19:1141-64.

24. Dietterich TG. Ensemble methods in machine learning; 2007.

25. Shu E, Ives Tallman C, Frye W, Boyajian JG, Farshidpour L, Young M, et al. Prehospital qSOFA as a predictor of sepsis and mortality. Am J Emerg Med. 2019; 37(7):1273-8. https://doi.org/10.1016/j.ajem.2018.09.025. Epub 2018 Sep 18.

26. Buschhorn HM, Strout TD, Sholl JM, Baumann MR. Emergency medical services triage using the emergency severity index: is it reliable and valid? J Emerg Nurs. 2013;39:e55-63.

27. Leeies M, Ffrench C, Strome T, Weldon E, Bullard M, Grierson R. Prehospital application of the Canadian triage and acuity scale by emergency medical services. CJEM. 2017;19:26-31.

28. Sun G, Shook TL, Kay GL. Inappropriate Use of Bivariable Analysis to Screen Risk Factors for Use in Multivariable Analysis. J Clin Epidemiol. 1996;49(8): 907-16.

29. Bagley SC, White $\mathrm{H}$, Golomb BA. Logistic regression in the medical literature: standards for use and reporting, with particular attention to one medical domain. J Clin Epidemiol. 2001:54:979-85.

30. LeCun Y, Bengio Y, Hinton G. Deep learning. Nature. 2015;521:436-44.

31. Kwon J, Lee $Y$, Lee $Y$, Lee $S$, Park J. An algorithm based on deep learning for predicting in-hospital cardiac arrest. J Am Heart Assoc. 2018;7:e008678.

32. Wolpert $\mathrm{DH}$, Macready WG. No free lunch theorems for optimization. IEEE Trans Evol Comput. 1997;1:67-82.

33. Christ M, Grossmann F, Winter D, Bingisser R, Platz E. Modern triage in the emergency department. Dtsch Arztebl Int. 2010;107:892-8.

34. Dugas AF, Kirsch TD, Toerper M, Korley F, Yenokyan G, France D, et al. An electronic emergency triage system to improve patient distribution by critical outcomes. J Emerg Med. 2016;50:910-8.

35. Checucci E, Autorino R, Cacciamani GE, Amparore D, De Cillis S, Piana A et al. Artificial intelligence and neural networks in urology: current clinical applications. Minerva Urol Nefrol. 2019. https://doi.org/10.23736/S0393-2249. 19.03613-0.

36. Chen X, Duan Y, Houthooft R, Schulman J, Sutskever I, Abbeel P. InfoGAN: Interpretable Representation Learning by Information Maximizing Generative Adversarial Nets; 2016.

37. Fong RC, Vedaldi A. Interpretable Explanations of Black Boxes by Meaningful Perturbation. In: Proc IEEE Int Conf Comput Vis 2017; 2017. p. 3449-57.

\section{Publisher's Note}

Springer Nature remains neutral with regard to jurisdictional claims in published maps and institutional affiliations.

Ready to submit your research? Choose BMC and benefit from:

- fast, convenient online submission

- thorough peer review by experienced researchers in your field

- rapid publication on acceptance

- support for research data, including large and complex data types

- gold Open Access which fosters wider collaboration and increased citations

- maximum visibility for your research: over $100 \mathrm{M}$ website views per year

At $\mathrm{BMC}$, research is always in progress.

Learn more biomedcentral.com/submission 